\title{
Richness of Thalloid Liverworts in Bilaspur, Achanakmar- Amarkantak Biosphere Reserve and Lafa hills, Chhattisgarh (India)
}

\author{
Ashwini Kumar Dixit \& Mery Aradhna Kerketta* \\ Department of Botany, Guru Ghasidas Vishwavidyalaya, Bilaspur 495 001, India
}

\section{Article history}

Received: 25 December 2018 Accepted: 06 April 2019

Published: 08 July 2019

\section{Publisher}

Horizon e-Publishing Group

\section{*Correspondence}

Mery Aradhna Kerketta

\mery.aradhna27@gmail.com

\begin{abstract}
A survey of bryophyte diversity in Bilaspur District (Chhattisgarh) and nearby areas has brought to light an unexpectedly rich bryoflora. Liverworts have a great diversity which includes both leafy and thalloid forms. Presence of 24 species of thalloid liverworts was recorded collectively from protected area Achanakmar-Amarkantak Biosphere reserve (AABR) as well as other regions of Bilaspur district and Lafa Hills, Korba. The main objective of this study is to evaluate the influence of elevation on the diversity of bryophytes in regional scale. For the study each location was mapped zone wise on 21 micro plots ranging between altitudes $230 \mathrm{~m}$ to $1011 \mathrm{~m}$. Using presence or absence of species, bryophyte species richness was compared at each altitude. These zones exhibit high species richness at mid-high elevation (around 525-1000 m) with an average 4-6 species of distribution rate. It is observed $83 \%$ of liverwort population as terrestrial, $10 \%$ as epiphytic, $7 \%$ grows in aquatic habitats and below $5 \%$ recorded as ubiquitous at various altitude ranges. Species composition shows variability along the elevation and microhabitat distribution which shows homogeneity of liverwort population. This study reveals the current status of liverworts in Bilaspur region.
\end{abstract}

Keywords: Thalloid Liverworts; Species distribution; Altitude; Diversity; Central India.

Citation: Dixit AK, Kerketta MA. Richness of Thalloid Liverworts in Bilaspur, AchanakmarAmarkantak Biosphere Reserve and Lafa hills, Chhattisgarh (India). Plant Science Today 2019; 6(3):287-298. https://doi.org/10.14719/pst.2019.6.3.469

Copyright: (c) Dixit \& Kerketta (2019). This is an open-access article distributed under the terms of the Creative Commons Attribution License, which permits unrestricted use, distribution, and reproduction in any medium, provided the original author and source are credited (https://creativecommons.org/licenses/by/4.0/).

Indexing: Plant Science Today is covered by Scopus,Web of Science, BIOSIS Previews, ESCI, CAS, AGRIS, CABI, Google Scholar, etc. Full list at http://www.plantsciencetoday.online

\section{Introduction}

Bilaspur, Korba and Achanakmar-Amarkantak Biosphere Reserve are part of biodiversity and also home of immense life forms including bryophytes. Bilaspur district is located in the western part of the state Chhattisgarh and lies between $21^{\circ} 37^{\prime}$ to $23^{\circ} 7^{\prime} \mathrm{N}$ latitudes and $81^{\circ} 12^{\prime}$ to $83^{\circ} 40^{\prime}$ E longitudes, which reside in Deccan peninsular Biogeographic zone and covered by Tropical deciduous forests which is extended to Achanakmar-Amarkantak Biosphere reserve (AABR) at North West and Lafa hills toward North 
and supported by different river system viz. Arpa and Shivnath rivers. Its topography ranging from high mountains, shallow valleys and plains, which provide suitable environment for the growth of hepatics. Although, there are reports of exploration and investigation on Bryophytes from Amarkantak-Achanakmar and other parts of central India (1-3), yet our knowledge about distribution of Hepatics in other regions of Chhattisgarh is quite meagre. Revised study on Achanakmar-Amarkantak Biosphere reserve by TFRI Jabalpur $(2,4-6)$ reported 69 species of bryophytes, out of which 17 are liverworts.

Considerable work on morphotaxonomical studies on liverworts has been done in India (7-11). Contribution from South-India (12-14) reported second largest population of liverwort species. In hotspot regions of Eastern Himalayas (15) and central India have substantial works $(1-3,5,16,17)$ published. However, the bryophyte diversity in this region is not yet fully understood.

Present study is an attempt to survey the unexplored areas in various ecological niches of liverworts from Chhattisgarh. Therefore the present communication deals with the assessment of diversity and distribution patterns of liverworts in the study area.

\section{Material and Methods}

For the assessment of species distribution in the region, random sampling has been done and patches are observed at each location. Then localities are categorized in three zones geographically. These locations were traced by GPS device (Gramin Montana 680). Twenty one sites were selected in and around Bilaspur region. Including Biosphere Reserve from Lafa hill range northeast Korba for permanent plots both observational and $10 \mathrm{~m}$ transect sites were selected based on characteristic species and associated microhabitats (Tables 1 \& 2). The effects of altitude and microhabitats on bryophyte diversity were parametrically tested with regression analysis. The parameters are expressed as altitude and microhabitats. The relationship between species assemblage and microhabitat were determined by Pearson correlation (18, 19); all data were compared using software package (SPSS 16.0v). The fresh specimens were collected from various natural localities of Bilaspur district and nearby areas of Chhattisgarh state (Fig. 1). All bryophyte species were collected and identified in the laboratory with the aid of a Leica microscope (DM2000) for morphological characterisation and available literature. These samples were deposited to Herbarium, Department of Botany, Guru Ghasidas Viswavidhyalaya, Bilaspur (Plates 1, 2 and 3).

\section{Enumeration of Taxa}

Family - Aytoniaceae

Asterella wallichiana (Lehm. \& Lindenb.) Grolle, Khumbu Himal. 1(4): 262. 1966.

Specimen examined: Central India, Chhattishgarh, Chaiturgarh; lat: 22.30.42.186 long: 82.16.15.108 alt: 772; 03.11.2016; M. Aradhna 0111109 (GGV: BOT).

Habitat- Rocks and walls, sometimes rheophytic.

Distribution- India, Sikkim, Assam, Western Ghats: Tamil Nadu, Maharashtra (17, 20, 21).

Asterella khasyana (Griff.) Grolle, Khumbu Himal. 1(4): 267. 1966; D.G. Long, Bryophyt. Biblioth. 63: 169. 2006.

Specimen examined: Central India, Chhattisgarh, Shivtarai (AABR) lat: 22.24.17.855 long: 81.52.7.8900 alt: 433, 07.02.2015; M. Aradhna 0110117 (GGV:BOT).

Habitat- Terricolus, in association with leafy Liverworts.

Distribution- India, Western Himalaya: Himachal Pradesh, Uttarakhand; Eastern Himalaya: Sikkim, Assam, Manipur, Meghalaya; Punjab, Rajasthan; Central India: Madhya Pradesh (Pachmarhi); Western Ghats: Tamil Nadu Karnataka, Maharashtra $(7,17,21,22)$.

Mannia indica Kachroo in J. Hattori Bot. Lab. 19:4. 1958. Grimaldia indica Steph., Sp. Hept. 6: 10. 1917.

Specimen examined: Central India, Chhattisgarh, location- Chaiturgarh (Jemra) lat: 22.31.28.5120 long: 82.14.41.574 alt; 543, 05-12-2014, M. Aradhna 01100441 (GGV:BOT).

Habitat- Terricolus along the road side rock. Association Plagiochasma sp.

Distribution- India, Western Himalaya: Himachal Pradesh, Uttarakhand; Eastern Himalaya: Sikkim, Assam, Nagaland; Punjab, Rajasthan; Central India: Western Ghats: Maharashtra $(7,17,22)$.

Plagiochasma intermedium Lindenb. \& Gottsche, Syn. Hepat. 513. 1846.

Specimen examined: Central India, Chhattisgarh, Chaiturgarh Lafa hills lat: 22.31.28.51200 long: 82.14.41.5740 alt: 543, 6-12-20-2014; M. Aradhna 0101125 (GGV:BOT).

Habitat- On the Rocks and cliff.

Distribution- India Western Himalaya: Himachal Pradesh, Uttarakhand; Punjab, Rajasthan, Uttar Pradesh; Western Ghats: Maharashtra $(12,17,22)$. 
Plagiochasma appendiculatum Lehm. \& Lindenb. in Nov. Strip. Pug. 4: 14. 1832.

Specimen examined: Central India, Chhattisgarh, location: Kabir chabutra lat: 22.40.30.101 long: 81.43.38.628 alt: 1009, 07.02.2015; M. Aradhna 01100019a-b (GGV:BOT).

Habitat- Terricolus, saxicolous around water bodies.

Distribution- India Western Himalaya: Himachal Pradesh, Uttarakhand; Eastern Himalaya: Sikkim, Assam, Manipur, Bengal (Darjeeling); Punjab, Rajasthan, Uttar Pradesh; Central India: Madhya Pradesh (Pachmarhi); Western Ghats: Tamil Nadu, Maharashtra, and Kerala (12, 13, 17, 22).

\section{Family - Cyathodiaceae K. Mull.}

Cyathodium denticulatum Udar \& S.C. Srivast. in Geophytology, 1(2): 166. 1971.

Specimen examined: Central India, Chhattisgarh, Chhaprwa- (AABR) lat: 22.24.17.855 long:
81.52.7.8900 alt: 433, 27.12.2016, M. Aradhna 0110116 (GGV:BOT).

Habitat- Terricolus, on moist soil rocky surface roadside AABR.

Distribution- India Darjeeling, India (Eastern Himalaya) (23) Achanakmar - Amarkantak Biosphere Reserve Chhattisgarh (Central India).

Cyathodium cavernarum Kuntze, Nov. Strip. Pug. 6: 18. 1834.

Specimen examined: Central India, Chhattisgarh, Chhaprwa- (AABR) lat: 22.24.17.855 long: 81.52.7.8900 alt: 433, 27.12.2016, M. Aradhna 0110117-b -c (GGV:BOT).

Habitat- Terricolus, in association with leafy liverworts and Asterella sp. Common throughout the region.

Distribution-India Western Himalaya: Himachal Pradesh, Uttarakhand; Eastern Himalaya: Sikkim, Assam, Manipur, Meghalaya, Nagaland, West Bengal (Darjeeling); Punjab, Uttar Pradesh; Central

Table 1. Distribution and occurrences of Liverworts in the study area with different habitats

\begin{tabular}{|c|c|c|c|c|c|}
\hline Species name & Zone 1 & Zone 2 & Zone 3 & Habitat* & Microhabitat** \\
\hline Marchantia papillata Raddi & + & - & - & $\mathrm{UM}$ & SX \\
\hline Marchantia paleacea Bertol." & + & - & - & $\mathrm{T}$ & TC: SX \\
\hline Dumortiera hirsuta (Sw.) Nees ${ }^{\circ}$ & - & + & - & UM & Fresh water stream :on bark \\
\hline Asterella wallichiana (Lehm. \& Lindenb.) Grolle & + & ++ & +++ & $\mathrm{T}$ & TC: SX \\
\hline Asterella khasyana (Griff.) Grolle & + & - & - & $\mathrm{T}$ & TC \\
\hline Mannia indica Kachroo ${ }^{*}$ & - & + & - & $\mathrm{T}$ & $\mathrm{TC}$ \\
\hline Plagiochasma appendiculatum Lehm. \& Lindenb. & + & ++ & +++ & $\mathrm{T}$ & TC \\
\hline Plagiochasma intermedium Lindenb. \& Gottsche & + & ++ & - & $\mathrm{T}$ & TC \\
\hline Reboulia hemisphaerica (L.) Raddi ${ }^{\natural}$ & + & - & - & $\mathrm{T}$ & TC \\
\hline Conocephalum conicum (L.) Dumort. & + & - & - & $\mathrm{T}$ & TC \\
\hline Targionia hypophylla L. & ++ & ++ & + & $\mathrm{T}$ & $\mathrm{TC}$ \\
\hline Cyathodium cavernarum Kunze & + & ++ & - & $\mathrm{T}$ & TC \\
\hline Cyathodium denticulatum Udar \& S.C. Srivast." & + & - & - & $\mathrm{T}$ & TC \\
\hline Riccia billardierei Mont. \& Nees & - & + & + & $\mathrm{T}$ & TC \\
\hline Riccia cavernosa Hoffm." & - & + & + & $\mathrm{T}$ & TC \\
\hline Riccia huebeneriana Lindenb. & - & + & + & $\mathrm{T}$ & $\mathrm{TC}$ \\
\hline Riccia frostii Austin & + & - & + & A:E & Bog \\
\hline Riccia sorocarpa Bisch. & - & + & - & $\mathrm{T}$ & TC \\
\hline Riccia fluitans L." & + & - & - & A & Bog:fen \\
\hline Pellia sp." & - & + & - & $\mathrm{T}$ & TC:SX \\
\hline Blasia pusilla L. & + & - & - & $\mathrm{T}$ & TC \\
\hline Aneura pinguis ( L.) Dumort. & + & - & - & $\mathrm{T}$ & TC \\
\hline Riccardia levieri Schiffn. & - & + & - & $\mathrm{T}$ & TC: SX \\
\hline Pallavicinia lyellii (Hook.) Gray ${ }^{\star}$ & - & + & - & $\mathrm{UM}$ & Fresh water stream: SX:TC \\
\hline
\end{tabular}


India: Madhya Pradesh (Pachmarhi); Western Ghats: Tamil Nadu, Karnataka, Maharashtra (7, 13, 17, 22).

\section{Family - Marchantiaceae}

Marchantia papillata Raddi subsp. grossibarba (Steph.) Bischl. in Cryptog. Bryol. Lichenol. 10: 78.1989. Marchantia grossibarba Steph. in Mem. Soc. Sci. Nat. Math. Cherbourg 29: 221. 1894.

Specimen examined: Central India, Chhattisgarh, Keonchi lat: 22.40.30.240 long: 81.43.39.131 alt: 887, 27.12.2016, M. Aradhna 0110016 (GGV:BOT).

Habitat- Saxicolus on steep rocks in with association of Notothylas sp. and Pheoceros sp.

Distribution- India Western Himalaya: Jammu \& Kashmir, Himachal Pradesh, Uttarakhand; Eastern Himalaya: West Bengal, Sikkim, Assam, Arunachal Pradesh, Meghalaya, Nagaland; Uttar Pradesh, Punjab, West Rajasthan, Central India: Madhya Pradesh (Pachmarhi); Western Ghats: Tamil Nadu $(7,12,13,17,22)$.
Marchantia paleacea Bertol. in Opusc. Sci. 1(4): 242. 1817; Bischl. In Bryophyt. Biblioth. 38:91. 1989. Marchantia nepalensis Lehm. \& Lindenb. in Nov. Strip. Pug. 4: 10. 1832.

Specimen examined: Central India, Chhattisgarh, Khodri, lat: 22.24.06.122 long: 81.52.7.8900 alt: 433, 27.12.2016, M. Aradhna 01101644 a, b (GGV: BOT).

Habitat- Terricolus, on the soil in association with Aneura sp. and Anthoceros sp. At shady moist derange places.

Distribution- India Western Himalaya: Jammu \& Kashmir, Himachal Pradesh; Eastern Himalaya: West Bengal, Sikkim, Assam, Uttar Pradesh; Central India: Madhya Pradesh (Pachmarhi); Western Ghats: Tamil Nadu (6, 7, 17, 22).

Dumortiera hirsuta (Sw.) Nees, Fl. Bras. Enum. Pl. 1: 307. 1833. Marchantia hirsuta Sw., Prodr. 145.1788 .

Specimen examined: Central India, Chhattisgarh, Kendai falls, Korba region Husdev river lat: 22.14.

Table 2. Sample sites with brief description GPS and Altitude

\begin{tabular}{|c|c|c|c|c|}
\hline Zonation & Location & Latitude & Longitude & Altitude \\
\hline \multirow{8}{*}{ Zone 1} & Dhudhadahara & $22^{\circ} 42^{\prime} 6.68^{\prime \prime} \mathrm{N}$ & $81^{\circ} 42^{\prime} 14.47^{\prime \prime} \mathrm{E}$ & 1008 \\
\hline & Kabir chabutra & $22^{\circ} 40^{\prime} 30.10^{\prime \prime} \mathrm{N}$ & $81^{\circ} 43^{\prime} 38.62 " \mathrm{E}$ & 1009.9 \\
\hline & Keonchi & $22^{\circ} 40^{\prime} 30.24^{\prime \prime} \mathrm{N}$ & $81^{\circ} 43^{\prime} 39.13^{\prime \prime} \mathrm{E}$ & 887 \\
\hline & Gurella Ghats & $22^{\circ} 34^{\prime} 60.47^{\prime \prime N}$ & $82^{\circ} 04^{\prime} 13.81^{\prime \prime} \mathrm{E}$ & 574.9 \\
\hline & Achanakmar (Shivtarai) & $22^{\circ} 24^{\prime} 17.85^{\prime \prime N}$ & 8152'07.89"E & 433 \\
\hline & Achanakmar (Amadob) & $22^{\circ} 31^{\prime} 52.95^{\prime \prime N} \mathrm{~N}$ & $81^{\circ} 44^{\prime} 52.12^{\prime \prime E}$ & 532 \\
\hline & Achanakmar (Bichoghati) & $22^{\circ} 29^{\prime} 16.41 " \mathrm{~N}$ & $81^{\circ} 47^{\prime} 24.94 " \mathrm{E}$ & 437 \\
\hline & Amarkantak (Maikunala) & $22^{\circ} 42^{\prime} 5.87^{\prime \prime N}$ & $81^{\circ} 42^{\prime} 19.56^{\prime \prime} \mathrm{E}$ & 1008.1 \\
\hline \multirow{6}{*}{ Zone 2} & Rajgamarg & $22^{\circ} 22^{\prime} 45.49^{\prime \prime} \mathrm{N}$ & $82^{\circ} 47^{\prime} 48.81^{\prime \prime E}$ & 272 \\
\hline & Korba (Main city) & $22^{\circ} 23^{\prime} 26.36^{\prime \prime} \mathrm{N}$ & $82^{\circ} 45^{\prime} 15.00 " \mathrm{E}$ & 328.9 \\
\hline & Kusmunda Husdev river & $22^{\circ} 14^{\prime} 43.64 " \mathrm{~N}$ & $82^{\circ} 39^{\prime} 42.81^{\prime \prime E}$ & 280 \\
\hline & Pali & $22^{\circ} 29^{\prime} 11.72 " \mathrm{~N}$ & $82^{\circ} 16^{\prime} 18.86^{\prime \prime} \mathrm{E}$ & 359 \\
\hline & Chaiturgarh (Jemra) & $22^{\circ} 31^{\prime} 28.51^{\prime \prime N}$ & $82^{\circ} 14^{\prime} 41.57 " \mathrm{E}$ & 543 \\
\hline & Chaiturgarh & $22^{\circ} 30^{\prime} 43.97 " \mathrm{~N}$ & $82^{\circ} 16^{\prime} 15.78^{\prime \prime E}$ & 862 \\
\hline \multirow{7}{*}{ Zone 3} & Bhanwar tonk & $22^{\circ} 36^{\prime} 33.40^{\prime \prime} \mathrm{N}$ & 8153'59.90"Е & 453.2 \\
\hline & Khodri (before Keonchi) & $22^{\circ} 32 ' 52.03 " \mathrm{~N}$ & $81^{\circ} 44^{\prime} 43.60^{\prime \prime} \mathrm{E}$ & 555.3 \\
\hline & Pendra & $22^{\circ} 37^{\prime} 41.78^{\prime \prime} \mathrm{N}$ & $81^{\circ} 43^{\prime} 39.89^{\prime \prime E}$ & 532 \\
\hline & Kenda & $22^{\circ} 30^{\prime} 13.46^{\prime \prime} \mathrm{N}$ & $82^{\circ} 41^{\prime} 00.63^{\prime \prime E}$ & 296.79 \\
\hline & Arpa river (Bilaspur) & $22^{\circ} 65^{\prime} 49.71 " \mathrm{~N}$ & $82^{\circ} 80^{\prime} 60.60^{\prime \prime E}$ & 244.9 \\
\hline & GGV campus & $22^{\circ} 07^{\prime} 30.55^{\prime \prime N}$ & $82^{\circ} 82^{\prime} 30.87^{\prime \prime E}$ & 282.5 \\
\hline & Dalhagiri & $22^{\circ} 55^{\prime} 40.31^{\prime \prime N}$ & $82^{\circ} 24^{\prime} 28.79^{\prime \prime} \mathrm{E}$ & 349.4 \\
\hline
\end{tabular}

Table 3. Summary of species distribution with elevation gradient statistical regression values

\begin{tabular}{lllll}
\hline Factors & $\boldsymbol{D} \boldsymbol{f}$ & $\boldsymbol{f}$ & $\boldsymbol{p}$ & $\mathbf{r}$ \\
\hline Species & 23 & 1.969 & $<0.001$ & 0.72 \\
\hline Elevation & 23 & 2.193 & $<0.05$ & 0.40 \\
\hline Habitat & 21 & 3.257 & $<0.05$ & 0.82 \\
\hline
\end{tabular}




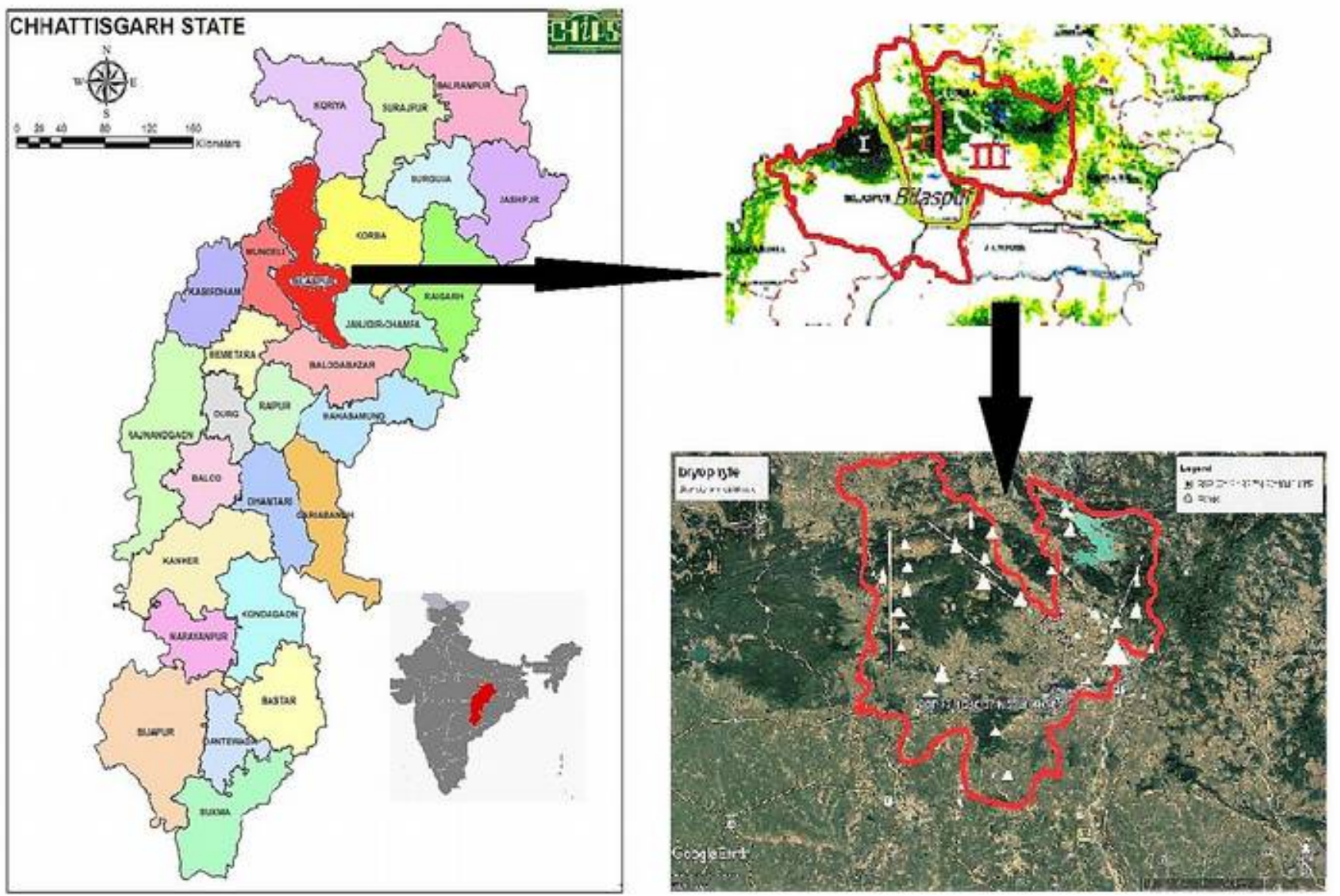

Fig. 1. Distribution of species in the study area, forest region around Bilaspur and Achanakmar-Amarkantak Biosphere Reserve, Chhattisgarh, India

43.649 long: 82.39.42.815 alt: 280, 16.10.2017; M. Aradhna 0111657 (GGV:BOT).

Habitat- On rocky surface in water falls and streams.

Distribution- India, Western Himalaya: Himachal Pradesh, Uttarakhand; Eastern Himalaya: West Bengal, Assam, Arunachal Pradesh, Meghalaya, Sikkim, Nagaland; Central India: Madhya Pradesh (Pachmarhi); Western Ghats: Tamil Nadu (6, 7, 17, 22).

\section{Family - Aneuraceae}

Aneura pinguis (L.) Dumort., Comment. Bot. 115. 1822. Jungermamia pinguis L., Sp. Pl. 2: 1136. 1753.

Specimen examined: Central India, Chhattisgarh, Maikunala (AABR) lat: 22.24.17.855 long: 81.52.7.8900 alt: 456, 07.02.2015; M. Aradhna 0110167 (GGV:BOT).

Habitat- Terricolus, on the soil in association with Marchantia sp. At shady moist places.

Distribution- India Western Himalaya: Himachal Pradesh, Uttarakhand; Eastern Himalaya: Sikkim, West Bengal (Darjeeling); Punjab, Rajasthan, Uttar Pradesh; Central India: Madhya Pradesh (Pachmarhi); Western Ghats: Tamil Nadu, Maharashtra (6, 7, 17, 22).
Riccardia levieri Schiffn. in Osterr. Bot. Z. 49: 130. 1899.

Specimen examined: Central India, Chhattisgarh, Chaiturgarh (Jemra) lat: 22.31.28.5120 long: 82.14.41.574 alt: 543, 05.07.2017; M. Aradhna 01101714 (GGV:BOT).

Habitat- Terricolus, on moist soil in association with Pheoceros sp.

Distribution- India, Western Himalaya: (Himachal Pradesh, Uttarakhand); Eastern Himalaya: West Bengal (Darjeeling); Central India: Madhya Pradesh (Pachmarhi); Western Ghats: Karnataka, Tamil Nadu (7, 17, 22).

\section{Family - Pallaviciniaceae}

Pallavicinia lyellii (Hook.) Gray, Nat. Arr. Brit. Pl. 1: 685, corr. 775. 1821. Jungermannia lyellii Hook., Brit. Jungermann. pl. 77. 1816.

Specimen examined: Central India, Chhattisgarh, Korba, Phool nadi , Rajga marg, lat: 22.22.45.49 long: 82.47.48.81 alt: 272, 06.12.2014; M. Aradhna 0110146 (GGV:BOT).

Habitat- On Rocky surface, rock cervices, fresh water stream.

Distribution- India, Eastern Himalaya (Assam), Central India (Madhya Pradesh - Pachmarhi), 


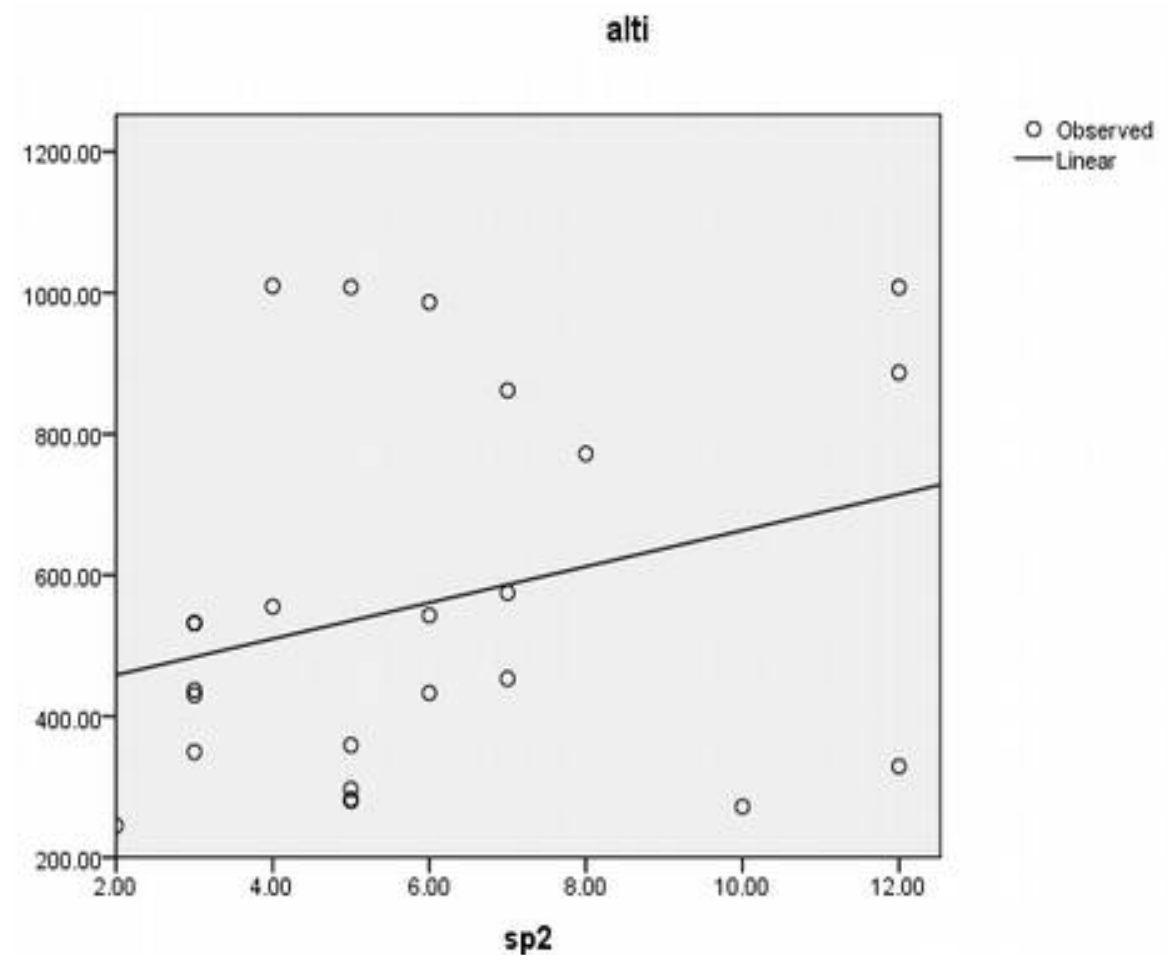

Fig. 2. a) Regression plot showing the relation between the altitude and distribution of species regression line were all significant $(\mathrm{p}<0.001) \mathrm{N}=24$

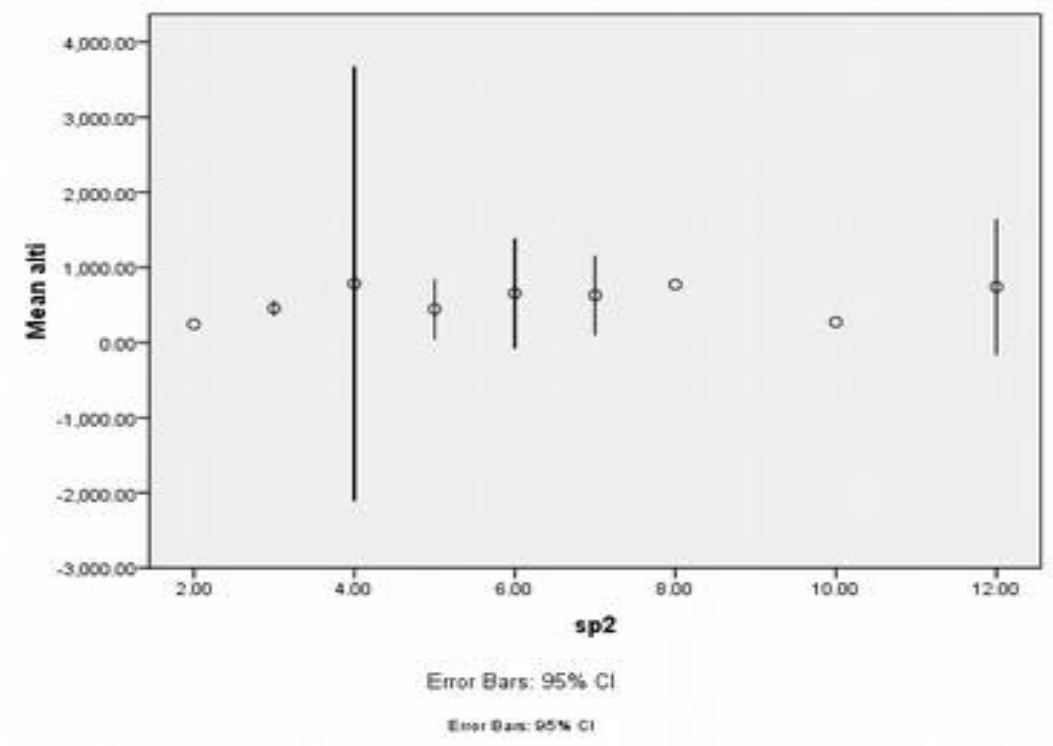

Fig. 2. b) Ordination graph for the canonical of correlation analysis of twenty one location with different micro habitat based on frequency of species within each site $(\mathrm{N}=21)$ with $95 \%$ CI, Mean elevation with average species distribution.

Western Ghats (Karnataka, Tamil Nadu) (6, 7, 17, 22).

\section{Family -Targioniaceae}

Targionia hypophylla L., Sp. Pl. 2: 1136. 1753.

Specimen examined: Central India, Chhattisgarh, Chhaprwa (AABR) lat: 22.24.17.855 long: 81.52.7.8900 alt: 433, 07.02.2015; M. Aradhna 0110199-a, b, c (GGV:BOT).

Habitat- Terricolus, on moist soil surface, Saxicolos in association with Mosses and Plagiocasma sp.

Distribution- India, Western Himalaya: Himachal Pradesh, Uttarakhand; Eastern Himalaya: Sikkim, Assam, Manipur, Meghalaya, West Bengal
(Darjeeling); Panjab, West Rajsthan, Uttar Pradesh; Central India: Madhya Pradesh (Pachmarhi); Western Ghats: Tamil Nadu, Karnataka, Maharashtra (6, 7, 17, 22).

\section{Family -Rebouliaceae}

Reboulia hemisphaerica (L.) Raddi, Opusc. Sci. 2: 357. 1818. Marchantia hemispherica L., Sp. Pl. 2: 1138.1753.

Specimen examined: Central India, Chhattisgarh, Amarkantak dhoodha-dhara lat: 22.42.6.68 long: 81.42.14.478 alt: 1008, 07.02.2015; M. Aradhna 0110193 (GGV:BOT).

Habitat- Terricolus on moist soil surface in association with leafy liverworts and rare in study area. 


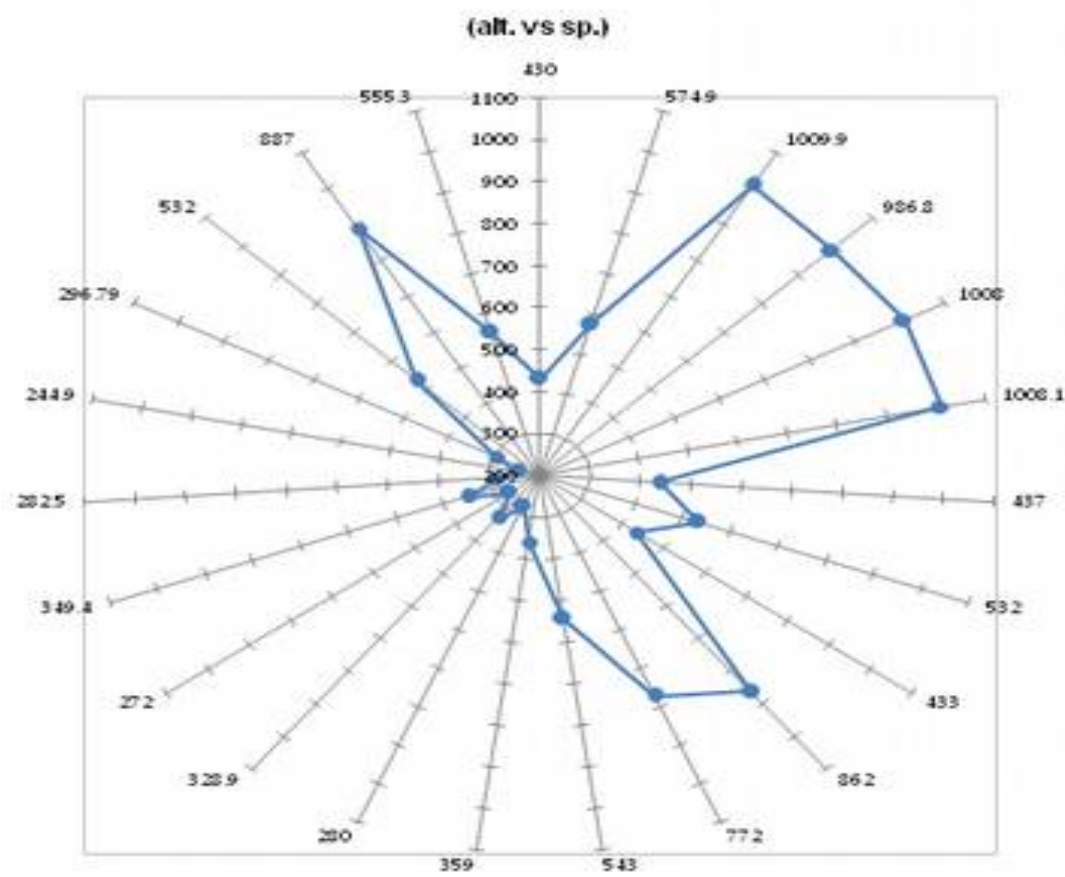

Fig. 3. Distribution of liverworts species in analogous elevation with maximum distribution (12-14 sp.) in range between $200 \mathrm{~m}-1010 \mathrm{~m}$.

Distribution- India, Western Himalaya: Himachal Pradesh, Uttarakhand; Eastern Himalaya: Sikkim, Assam, Manipur, Meghalaya, West Bengal (Darjeeling); Punjab, Rajasthan, Uttar Pradesh; Central India: Madhya Pradesh (Pachmarhi); Western Ghats: Tamil Nadu, Karnataka $(13,17,22)$.

\section{Family-Conocephalaceae}

Conocephalum conicum (L.) Dumort., Comment. Bot. 115. 1822. Marchantia conica L., Sp. pl. 2: 1138. 1753.

Specimen examined: Central India, Chhattisgarh: Shivtarai Bhichoghati (AABR) lat: 22.24.17.855 long: 81.52.7.8900 alt: 433, 23.10.2016; M. Aradhna 01100401 (GGV:BOT).

Habitat- Terrestrial near to stream in association with leafy Liverworts.

Distribution- India Western Himalaya: Jammu \& Kashmir, Himachal Pradesh, Uttarakhand; Eastern Himalaya: Sikkim, Assam, Manipur, Nagaland; Punjab, Rajasthan; Central India: Madhya Pradesh (Pachmarhi); Western Ghats: Kerala (7, 13, 17, 22).

\section{Family -Blasiaceae}

Blasia pusilla L., Sp. Pl. 2: 1138. 1753.

Specimen examined: Central India, Amarkantak lat: 22.42.5.87390 long: 81.52.7.8900 alt: 1008.1 lat: 22.24.17.855 long: 81.52 .7 .8900 alt: 433, 19.11.2017; M. Aradhna 01110114 (GGV:BOT).

Habitat- Terricolus, on moist soil associated with moss, Anthoceros sp.
Distribution- India, Western Himalaya: Himachal Pradesh, Uttarakhand; Eastern Himalaya: Assam $(7,17,22)$.

\section{Family - Ricciaceae}

Riccia frostii Austin in Bull. Torrey Bot. Club 6: 17. 1875.

Specimen examined: Central India, Chhattisgarh, Arpa river (Bilaspur) lat: 22.65.49.7190 long:82.80.60.60 alt: 244.9, 09-09-2015; M. Aradhna 01101538 (GGV:BOT).

Habitat- Terricolus, bank of Arpa river along koni.

Distribution-India, Western Himalaya: Jammu \& Kashmir; Eastern Himalaya: Sikkim, Assam, Manipur, Gangetic plains: West Bengal, Punjab, Uttar Pradesh; Western Ghats: Tamil Nadu, Kerala $(7,17,22)$.

Riccia sorocarpa Bisch., Nova Acta Phys.-med. Acad. Caes. Leop. - Carol. Nat. Cur. 17: 1053. 1835.

Specimen examined: Central India, Chhattisgarh, Korba (Main city) lat: 22.23.26.0119 long: 82.45.15 alt: 328.9, 09-09-2015; M. Aradhna 01101533 (GGV: BOT).

Distribution - India Western Himalaya: Himachal Pradesh, Uttarakhand; Eastern Himalaya: West Bengal (10).

Riccia fluitans L., Sp. Pl. 2: 1139. 1753.

Specimen examined: Central India, Chhattisgarh, Kabir chabutra lat: 22.40.30.101 long: 81.43.38.628 alt: 1009, 05-03-2016; M. Aradhna 01100024 (GGV:BOT). 

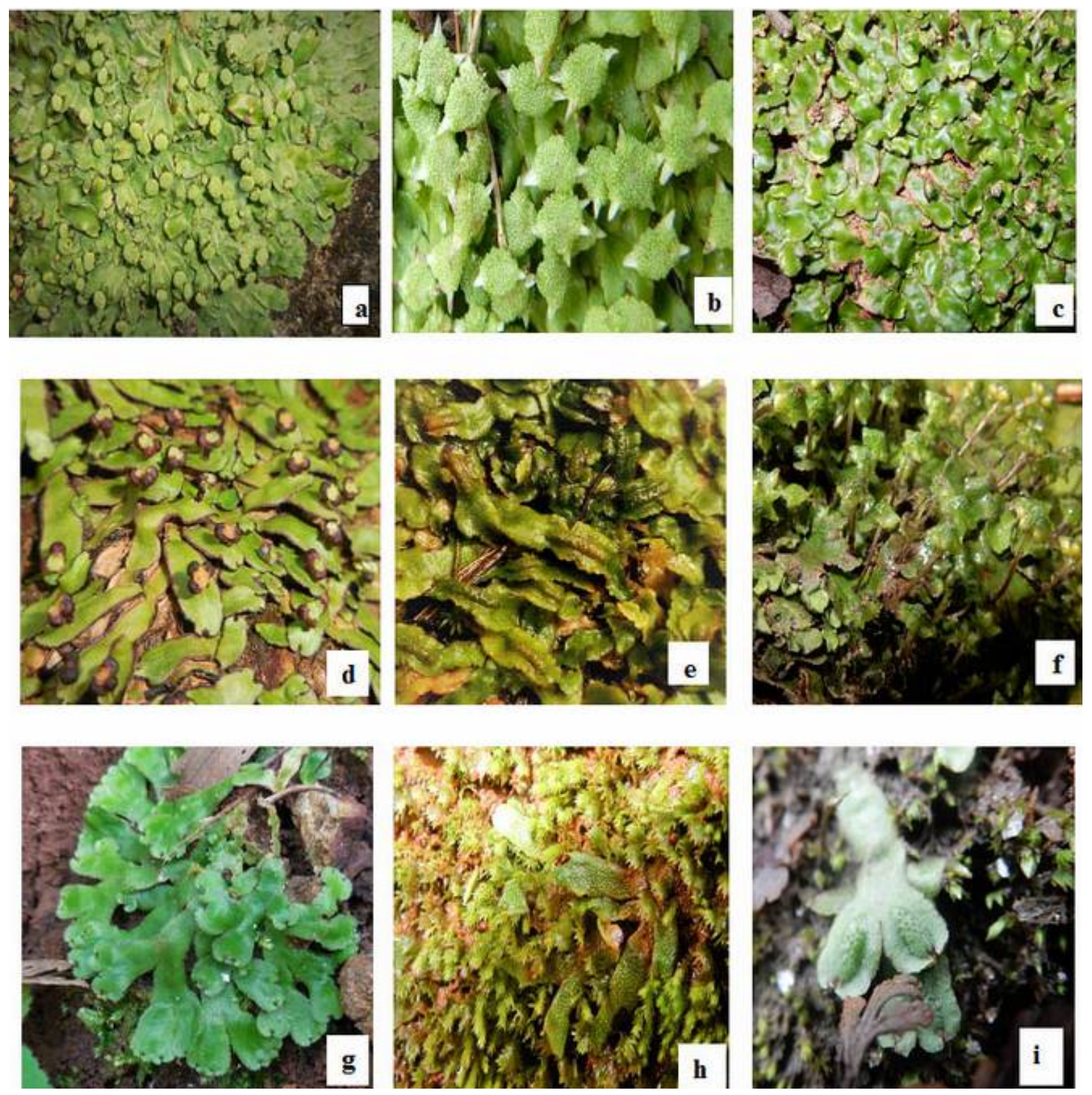

PHOTO PLATE I. a. Asterella khasyana (Griff.) Grolle b. Asterella wallichiana Lehm. c. Plagiochasma appendiculatum Lehm. \& Lindenb. d. P. intermedium lindenb. \& Gottsche e. Mannia indica (Steph.) Kachroo f-g. Reboulia hemisphaerica (L.) Raddi h-i. Conocephalum conicum (L.) Dumort.

Habitat- Aquatic, in association with other aquatic plants.

Distribution- India, Western Ghats: Karnataka (10, 13, 17).

Riccia billardierei Mont. \& Nees, Syn. Hept. 602. 1846.

Specimen examined: Central India, Chhattisgarh, Shivtarai (AABR) lat: 22.24.17.855 long: 81.52.7.8900 alt: 433, 20-09-2016; M. Aradhna 0110302 (GGV: BOT).
Habitat- Terricolus, grows in moist places in association of other Riccia spp.; common throughout the region.

Distribution- India Western Himalaya: Himachal Pradesh, Uttarakhand; Eastern Himalaya: Sikkim, Assam; Gangetic Plains: West Bengal-plains; Punjab, Uttar Pradesh, Bihar; Central India: Madhya Pradesh; Western Ghats: Karnataka, Maharashtra $(10,13,17)$.

Riccia cavernosa Hoffm., Deutschl. Fl. 2: 95. 1796 emend. Raddi, Opusc. Sci. (Bologna) 2: 351.1818.

Specimen examined: Central India, Chhattisgarh, Guru Ghasidas Viswavidyalaya campus lake lat: 

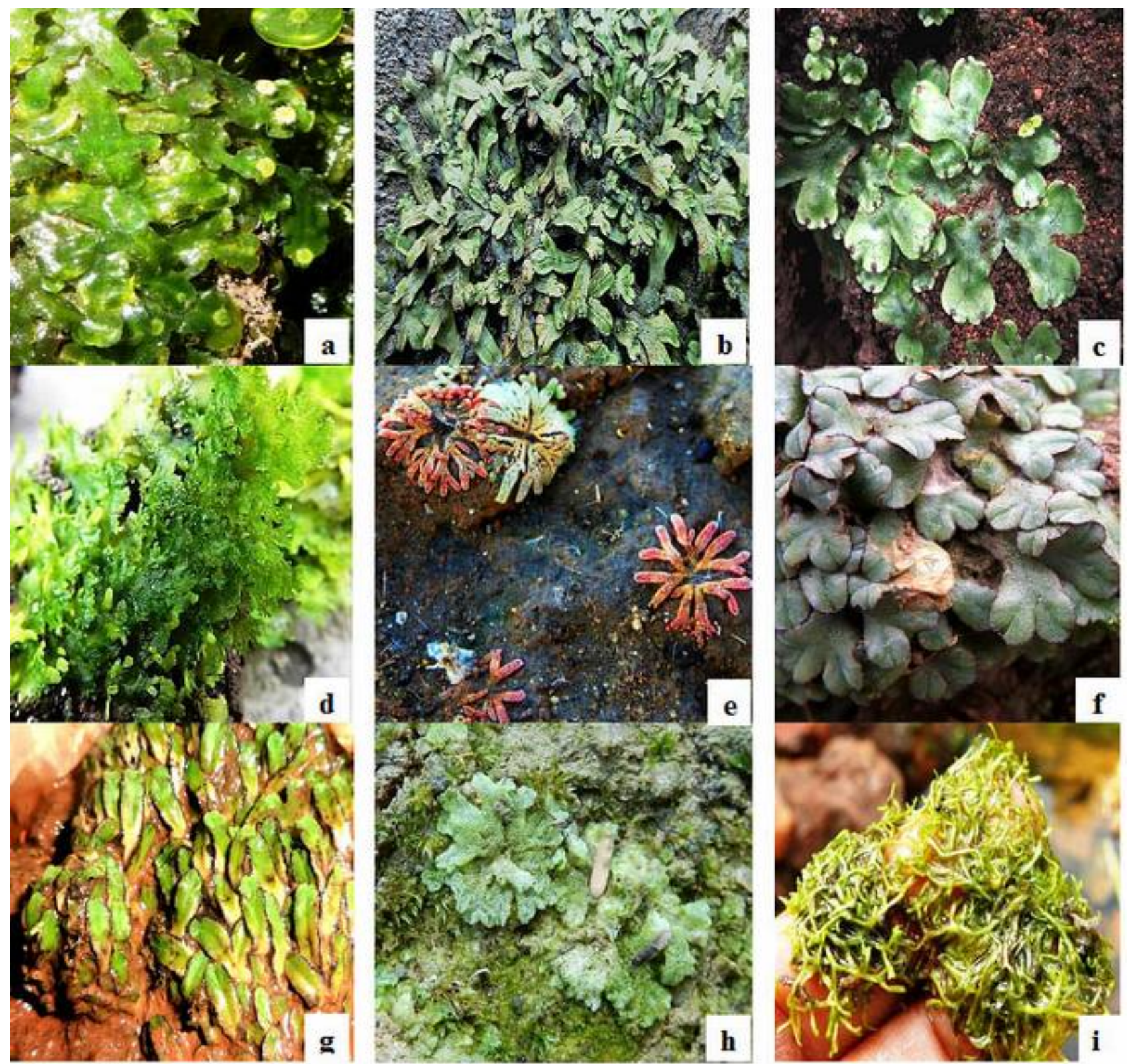

PLATE II. a. Dumortiera hirsuta (Sw.) Nees b. Marchantia papillata subsp. grossibarba (Steph.) Bischl. c. Marchantia paleacea Bertol. d. Riccardia levieri Schiffn. e. Riccia frostii Austin f. Riccia sorocarpa Bisch. g. Riccia billardierei Mont. \& Nees h. Riccia cavernosa Hoffm i. Riccia fluitans.

22.7.30.551900 long: 82.823 .08769 alt: 282.5 , 02.03.2016; M. Aradhna 0111625 (GGV:BOT).

Habitat- Terricolus, very specifically growing on bank of water bodies in and around (GGV) university near (BOT).

Distribution- India, Western Himalaya: Himachal Pradesh; Eastern Himalaya: West Bengal Hills; Uttar Pradesh $(10,13,17)$.

Riccia huebeneriana Lindenb. in Nov. Actorum Acad. Caes. Leop.-Carol. German. Nat. Cur. 18: 504. 1837.

Specimen examined: Central India, Chhattisgarh, Chaiturgarh (jemra) lat: 22.31.28.51200 long: 82.14.41.5740 alt: 543, 23.10.2017; M. Aradhna 01101729 (GGV:BOT).
Habitat- Terricolus, side of paddy fields.

Distribution- India, Eastern Himalaya: Sikkim, Assam, West Bengal; Central India: Madhya Pradesh (Pachmarhi); Western Ghats: Karnataka $(7,10,13,17)$.

\section{Family - Pelliaceae}

Pellia endiviifolia (Dicks.) Dumort., Recuiel Observ. Jungerm. 27. 1835.

Specimen examined: Central India, Chhattisgarh, Shivtarai (AABR) location; Amarnath Caves Chaiturgarh Palli lat: 22.30.43.0979 long: 82.16.15.7800 alt: 862, 03-11-2016; M. Aradhna 3611125 (GGV:BOT).

Habitat- In moist rock crevices, rare in study region. 

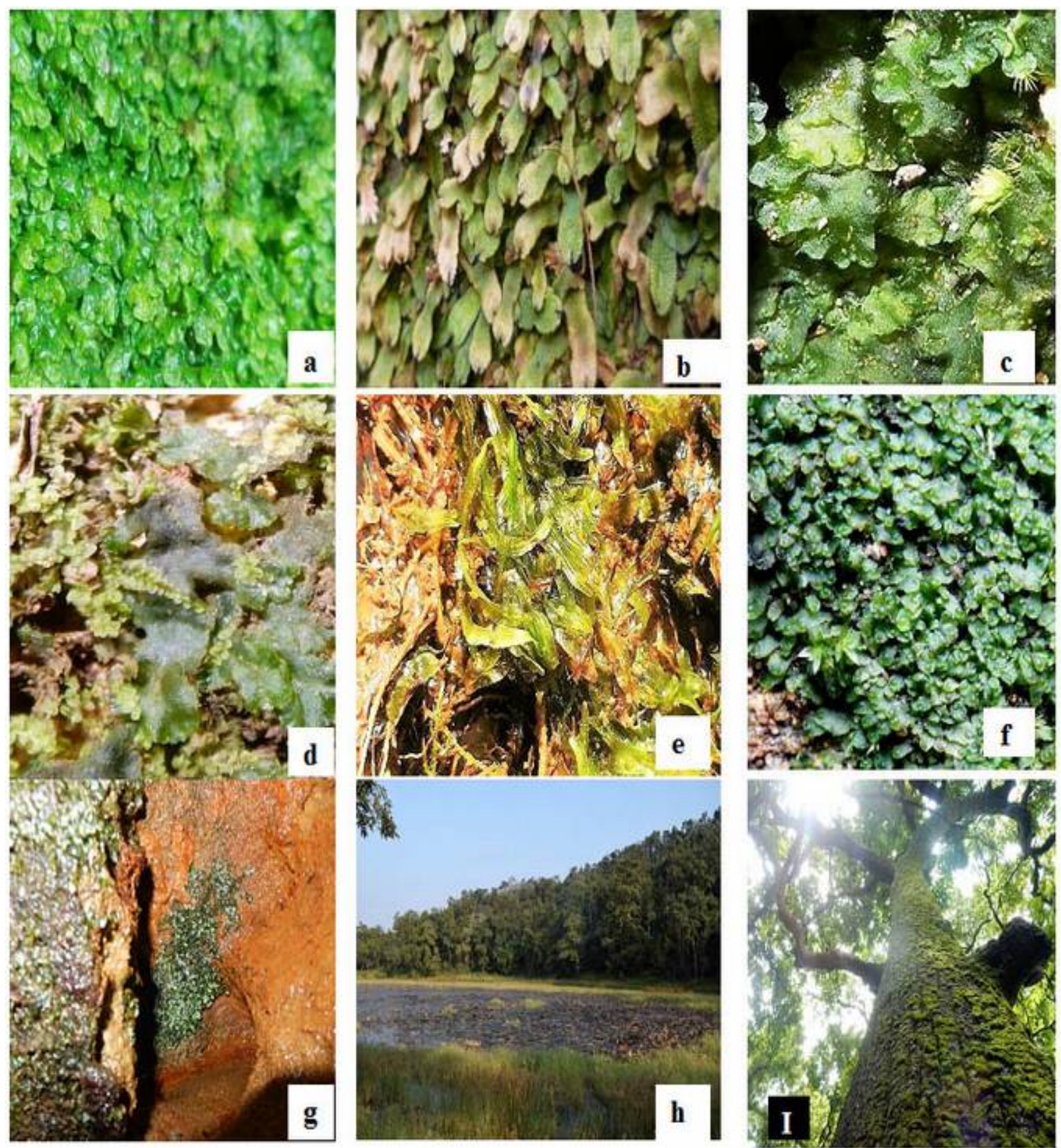

Plate III. a. Cyathodium cavernarum Kunze b. Targionia hypophylla L. c. Blasiapu silla L. d. Aneura pinguis (L.) Dumort. e. Pallavicinia lyellii (Hook.) Gray f. Pellia sp., different habitats g. Pellia sp. growing in rock crevices h. Fen i. epiphytic substratum.

Distribution- Eastern Himalaya: Sikkim, Assam, West Bengal; Western Ghats: Karnataka $(13,17)$.

\section{Result and Discussion}

\section{Species Richness}

Plants were collected from micro-plots and identified to species level. The parameters are expressed as altitude and microhabitat, for study distribution in a geographical area. Correlations determined by calculating the Pearson coefficient for mean distributed values at $P<0.01$ was considered to indicate statistical significance. The data obtained through different statically analysis shows monotonic distribution pattern of species according to altitude (Regression: Species gradient; $\mathrm{R}=0.72, d f=23, f=1.96, P<0.01)$. The function obtained for the two variables compared for all species i.e. mean altitude, species occurrence. The mean altitude derived as $595 \mathrm{~m}$, the highest similarities found between altitudes 850 to $1010 \mathrm{~m}$ with minimum 6-8 species and maximum no of species 12-14 per location, while the altitudinal range between 440 to $750 \mathrm{~m}$ exhibited the lowest 
similarity 3 to 8 species at various locations (Fig. $3)$.

The value plotted and fitted for mean value is contrasting between the different locations (alt. Range 230-1011 m). It has been observed that the abundance at lower altitudes between $230 \mathrm{~m}$ to $400 \mathrm{~m}$ also bring the same pattern of richness as higher altitude with min 4-5 and maximum 8-12 species at different sites. (Regression: Elevation gradient; $\mathrm{R}=0.40, d f=23, f=2.193, P<0.05)$. Regression drawn at significant level $(\mathrm{p}<0.05)$; the range of altitude variation (min $224.90 \mathrm{~m}-\max$ $1009.90 \mathrm{~m}$ ) mean elevation $595.3 \mathrm{~m}$ (altitude) correlation coefficient $0.82 ; \mathrm{R}^{2} 72 \%$ (Fig. 2, Table 3). Local richness was significantly influenced by altitude. Bryophyte species richness increased along the altitudinal gradient. During the field study four to eight species were present per location as in patches; statistical analysis showed distribution of liverworts with an average of four species per location (Fig. 2b).

\section{Distribution}

Habitats partitioning are superficially based on niche segregation. Each substratum observed individually (moist soil, rocky crevices, clefts, turf, exposed condition, shady places, epiphytic, bark, other plant substrate, aquatic, bog, fens etc.). These are then classified in to three major groups terrestrial, aquatic and epiphytic. Out of 24 taxa identified $83 \%$ have been found growing as terrestrial, $5 \%$ as strictly epiphytes, $5 \%$ terrestrial as well as epiphytic while $7 \%$ are aquatic.

Out of 24 species, 10 species recorded only once and these are specific to certain habitats (marked with "in Table 1). Rest of the species showed scattered distribution according to elevation gradient. (Habitat gradient; $\mathrm{R}=0.82, d f=$ $21, f=3.257, P<0.05)$. The highest similarity found between ranges (200-450) and (800-1009). The number of species shared vastly varied 3-14 between each altitude, at lower altitude species richness was greater on ground but as altitude increases species richness on other substrate was also observed, but that was less distinct. Overall ground microhabitat is occupied at every altitude, which shows homogeneity of thalloid liverworts exists within microhabitats or different substrates.

\section{Floristics}

Twelve species of thalloid liverworts appeared to be previously reported from Chhattisgarh (4). A total of 24 species of thalloid liverwort were reported, that includes 14 genera belonging 11 families. Thalloid liverworts viz., Asterella wallichiana Lehm., Mannia indica (Steph.) Kachroo, Plasiochasma intermedium linden. \& Gottsche, Reboulia hemisphaerica (L.) Raddi, Conocephalum conicum (L.) Dumort., Cyathodium cavernarum Kunze L., C. denticulatum Udar et Srivastava, Riccia cavernosa Hoffm, $R$. huebeneriana Lindenb., $R$. sorocarpa Bisch., $R$. flutians L., $R$. frostii Austin and Pallavicinia lyellii (Hook.) Gray are new record for the state.

Among these 24 thalloid liverworts, 5 species are contagiously distributed, which accounted more than $30 \%$ and belong to family Aytoniaceae, Cyathodiaceae and Targioniaceae viz. Plagiochasma appendiculatum Lehm. \& Lindenb., $P$. intermedium Linden. \& Gottsch, $A$. wallichiana Lehm, Targionia hypophylla L., Cyathodium sp. These taxa were adapted to arid climate (xeromorphic) and distributed abundantly throughout region. Regionally rare species recorded i.e. species occurring less than of $10 \%$ from the 21 different locations. Four species are very rare and site specific viz. Dumortiera hirsuta (Sw.) Nees, Riccia hemisphaerica (L.) Raddi, Pallavicinia lyellii (Hook.) Gray and Cyathodium denticulatum Udar \& S.C. Srivast.

It is concluded from data that species show significant result with richness increases as altitude rises; it is also observed at lower altitudes dense microhabitats such as rocky crevices and cave-like structures also provide substrate to grow successfully. The significance value with correlation high significant at 0.72 for species assemblage, and 0.82 for habitat distribution but altitude distribution shows moderate significance value 0.42 . Genera like Marchantia sp., Dumortiera hirsuta (Sw.) Nees, Pallavicinia lyellii (Hook.) Gray growing on wide range of substrate which helps them to escape disjunction. No comprehensive reports are available on the bryoflora diversity of this region. It is found from the study, that genera growing in this region have wide range of distribution and contributed a large percentage of total liverwort floras of Bilaspur, AABR and Lafa hills. This study is limited by the fact that only elevation and differential habitats were studied. Distribution mapping were carried out, this might be possible that other ecological factors and other group of bryophytes should also be studied for holistic approach to understand growth patterns. However, we found it worth reporting that this one dimensional studies on altitudinal gradient and habitat factors on distribution of liverworts which significantly resulted in comparison of richness. This comprehensive study also provides baseline information on the bryo-vegetation of Bilaspur region.

\section{Acknowledgements}

The authors are grateful to the UGC for financial assistance and to Bilaspur Regional Forest Department for providing facilities for exploration.

\section{Conflict of Interest}

The authors declared that they have no conflict of interest. 


\section{Author's Contribution}

All the authors contributed equally to the work presented in this paper.

\section{References}

1. NathV, Ashtana AK, Kapoor R. Enumeration of the Mosses in Amarkantak (Madhya Pradesh), India-I. Taiwania, 2007;52(2):168-76. https://doi.org/10.6165/tai.2007.52(2).168

2. Kapoor R, Nath V, Asthana A K. Bryophytes of Kanha National Park (Madhya Pradesh) India Nelumbo, 2011;53:140-144

3. Gupta R, Nath V, Asthana A K. Present scenario of Moss diversity at tamia hills and Patalkot valley (Madhya Pradesh) India. National Academy Science letters https://doi.org/10.1007/s40009-013-0182-7 2013;36(6):629-634

4. BRIS. Biosphere reserve information series 2014; 1:2: http://tfri.icfri.gov.in

5. Roychudhury N, Sharma R, Mishra RK. Achanakmar-Amarkantak Biosphere reserve India: A diverse tropical forest ecosystem. Vansangyan 2016 May vol. 3(5): 1-5.

6. Kapoor R, Nath V, Asthana A K. Studies on the bryoflora of Achanakmar wildlife sanctuary, Chhattishgarh, India. Geophytology.2008 38(1-2): 2535

7. Das S, Sharma GD. Inventorization of Marchantiophyta in Barail Wildlife Sanctuary, Assam, India with reference to their microhabitat. Archive for Bryology 2013:166;1-27. http://frahmia.de/downloads/archive of bryology/Ar chive\%20166.pdf

8. Singh DK. Diversity in Indian Liverworts: Their status, vulnerability and conservation. Nath V, Asthana AK, eds. Perspectives in Indian bryology. Bishen Singh Mahendra pal Singh, 2001; 35-53.

9. Singh DK, Singh SK. Diversity in Liverworts and Hornworts of Great Himalayan National Park, Western Himalaya, India. In Mohamed $\mathrm{H}$. et al (eds.), Bryology in the New Millennium University of Malaya. Kuala lumpur; 2008:291-317.

10. Singh SK, Bag AK, Bhattacharya SG. Riccia (Hepaticae: Ricciaceae) of west Bengal. Taiwania. 2010 jun 1; $55(2)$ : 109. https://doi.org/10.6165/tai.2010.55(2).99

11. Singh SK. An Appraisal of Genus Riccia in india with a note on Diversity and distribution of Species. Int J. Sustain Water and Environ Syst.2014: 6 (1):35-43.
12. Alam A, Srivastava SC. Current status of genus Plagiochasma in Nilgiri and Palani hills with SEM details of spores. Indian journal of Forestry 2009; 32(4): 623-634.

13. Daniels AED. Checklist of the Bryophytes of Tamil Nadu. India Archive for Bryology 2010:65;1-117. http://www.frahmia.de/downloads/archive of bryol ogy/Archive\%2065.pdf

14. Mishra M, Dash PK, Alam A, Sahoo S, Das R. Current status of diversity and distribution of Bryophytes of Odisha. Plant Science Today. 2016;3(2):186-194. https://doi.org/10.14719/pst.2016.3.2.222

15. Srivastava SC. Distribution of Hepaticae and Anthocerotae in India. Chopra RN, eds. Topics in Bryology. 1998:53-85.

16. Alam A, Rawat KK, Varma PK, Sharma V, Gupta DS. Moss Flora of Central India. Plant Science Today $2015 \quad$ Oct. $\quad 1$; $159-171$ https://doi.org/10.14719/pst.2015.2.4.126

17. Dandotiya DI, Govindapyari H, Suman SH, and Uniyal PL. Checklist of the Bryophytes of India. Arch Bryol. 2011; 88(1):126. http://www.archive-forbryology.com/Archive\%2088.pdf

18. Ah-Peng C. Chuah-Petiot M, Descamps Julien B. Bardat J. Stamenoff P, Strasberg D. Bryophyte Diversity and Distribution along an altitudinal gradient on a lava flow in LA Reunion. Diversity and distributions, 2007; $13 \quad$ (5): 654-662. https://doi.org/10.1111/j.1472-4642.2007.00393.x

19. Michael Kessler. The impact of population processes on patterns of species richness: lessons from elevation gradients. Basic and Applied Ecology 2009;10:295-299 https://doi.org/10.1016/j.baae.2008.10.006

20. Long DG. Revision of the genus Asterella P.Beauv. in Eurasia. Bryophytorum Bibliotheca, Band 63. 2006.

21. Naveen Sahu, Afroz Alam, Krishna Kumar Rawat, Praveen Kumar Verma. Spore morphology Of some south Indian species of Asterella P.Beauv. (Marchantiophyta) Am. Sc. 2014:10(4):18-24. http://www.jofamericanscience.org/journals/am-sci/ am1004s/003 23198am1004s14 18 24.pdf

22. Singh SK, Singh DK. A catalogue of the Liverworts and Hornworts of Himachal Pradesh, India. Univ.Bibliothek;2010.

23. Srivastava SC. Dixit R. The genus Cyathodium Kunze. Journal of The Hattori Botanical Laboratory; 1996.80:145-215 